\title{
Comment on "Prevalence and Cognitive Bases of Subjective Memory Complaints in Older Adults: Evidence from a Community Sample"
}

\author{
Ashima Nehra, Sakshi Chopra, and Harsimarpreet Kaur \\ Neurosciences Centre, All India Institute of Medical Sciences, Room No. NS-718, Ansari Nagar, New Delhi 110029, India \\ Correspondence should be addressed to Ashima Nehra; ashimanwadhawan@gmail.com
}

Received 8 July 2014; Accepted 24 July 2014; Published 13 August 2014

Academic Editor: Yaroslav Winter

Copyright (c) 2014 Ashima Nehra et al. This is an open access article distributed under the Creative Commons Attribution License, which permits unrestricted use, distribution, and reproduction in any medium, provided the original work is properly cited.

It was a pleasure reading your paper entitled "Prevalence and Cognitive Bases of Subjective Memory Complaints in Older Adults: Evidence from a Community Sample," which was published on 27 April 2014. It was a very detailed paper about the incidence of subjective memory complaints (SMCs) in older adults and how importantly SMCs could have a prognostic value in predicting objective memory decline and dementia $[1,2]$.

It was very interesting to note the process of making subjects as a part of the study, who are drawn from memory clinics, biased in finding a higher prevalence of SMC as subjects are more likely to have memory impairments, whereas, on the other hand, how population based studies may be biased in finding a lower prevalence of SMC's as the subjects are more willing to participate in research and are also healthier than the patient groups (subjects drawn from memory clinics).

However, in the methodology, the clear inclusion and exclusion criteria were not mentioned for the subjects who were made a part of the study. There was no mention about any comorbid conditions or disorders as well, which the participants who were included in the study had, as this information can be important for further having any correlations between the existing comorbid conditions/disorders and SMCs. Also, the subjects were divided into two groups, younger adults and older adults, but there was no mention on what criteria this was based. According to the World Health Organization, any person who is 60 years old and above can be called an elderly or an older population [3], so we would like to know why subjects who were 60 years old and above were not included and why 65 years old and above subjects were enrolled.

Moreover, in the methods, there were 3 hypotheses of the study, but there was no mention about them being accepted or rejected. The third objective which stated that gender and objective memory would be associated with a greater risk for SMCs in women than in men was rejected after obtaining the results. The same could have been stated in the results and conclusions.

The paper was also very comprehensive about the data and results, but nothing was mentioned about the subjects who actually performed low in the objective neuropsychological evaluation, who turned out to have dementia, whether they referred to a neurology clinic after that for any treatment/intervention or not. The future directions and implications of the study could have been highlighted and how the study can be benefitted to the general population to make substantial generalizations would further strengthen the research.

Overall, it was a very interesting study, and we hope that these can be done in India as well, soon.

\section{Conflict of Interests}

The authors declare that there is no conflict of interests regarding the publication of this paper. 


\section{References}

[1] L. Wang, G. Van Belle, P. K. Crane et al., "Subjective memory deterioration and future dementia in people aged 65 and older," Journal of the American Geriatrics Society, vol. 52, no. 12, pp. 2045-2051, 2004.

[2] A. F. Jorm, K. H. Masaki, H. Petrovitch, G. W. Ross, and L. R. White, "Cognitive deficits 3 to 6 years before dementia onset in a population sample: the Honolulu-Asia Aging Study," Journal of the American Geriatrics Society, vol. 53, no. 3, pp. 452-455, 2005.

[3] World Health Organization, Definition of an Older or Elderly Person, Geneva, Switzerland, 2009. 


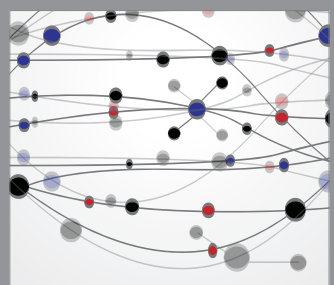

The Scientific World Journal
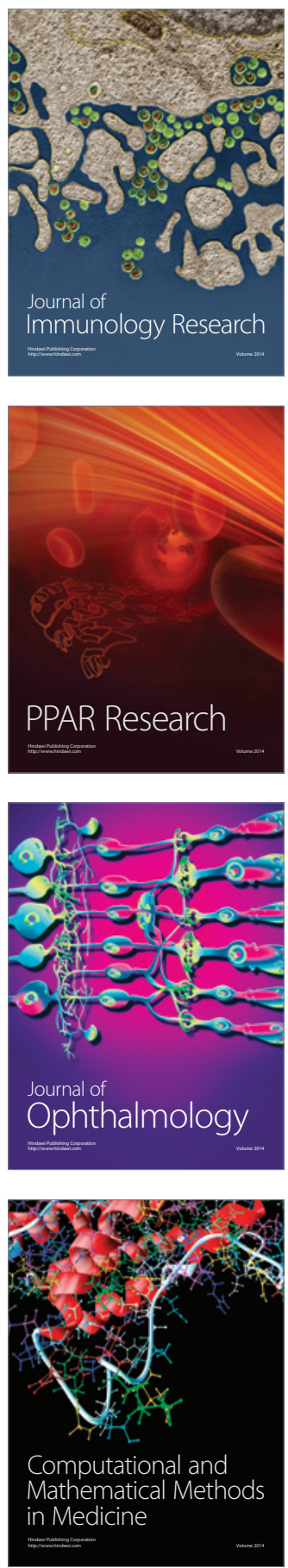

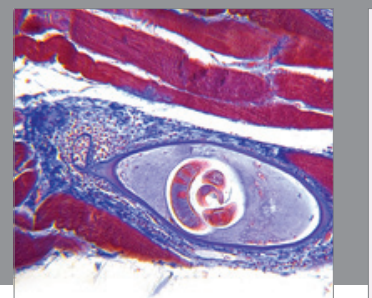

Gastroenterology

Research and Practice
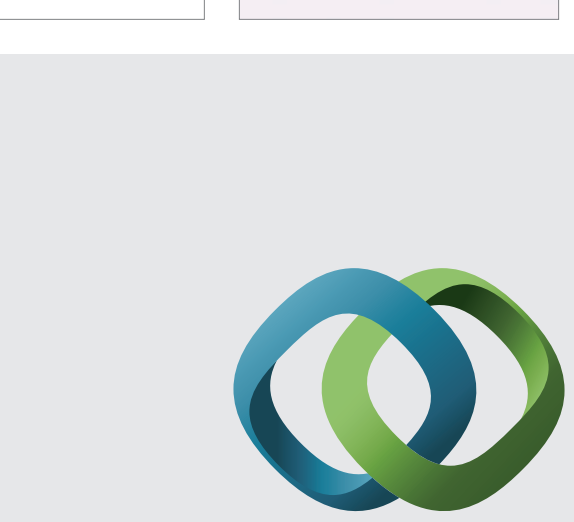

\section{Hindawi}

Submit your manuscripts at

http://www.hindawi.com
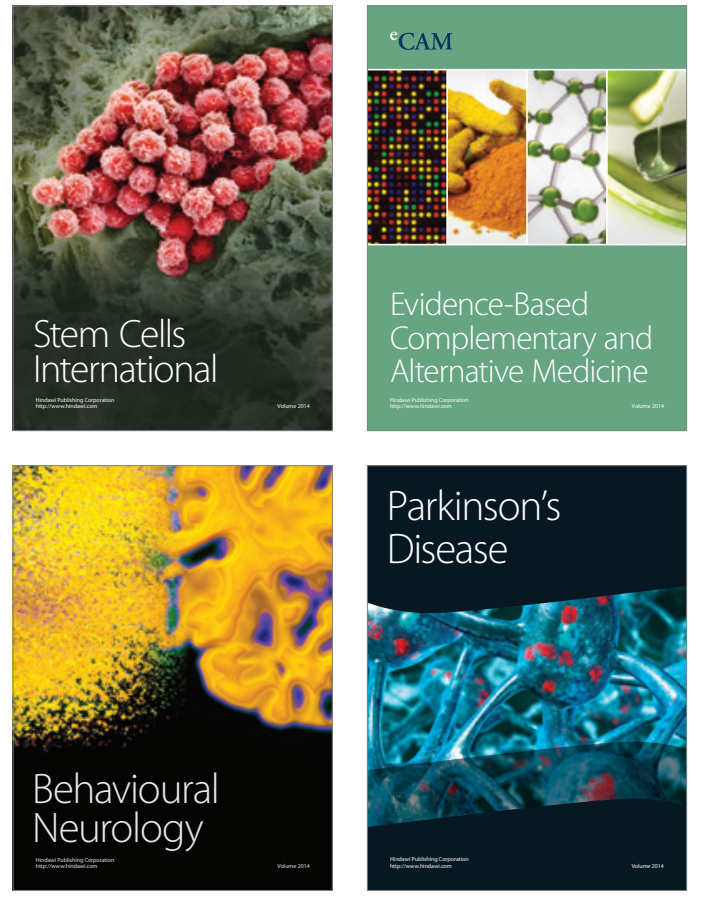
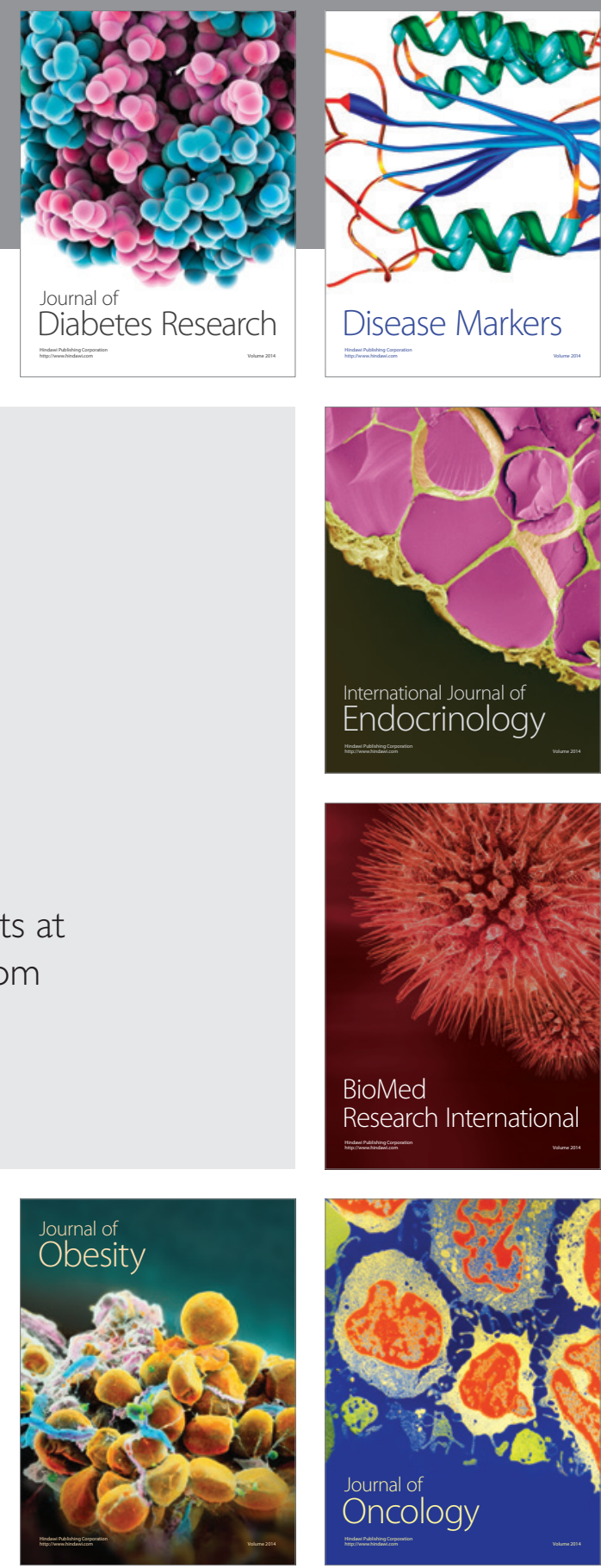

Disease Markers
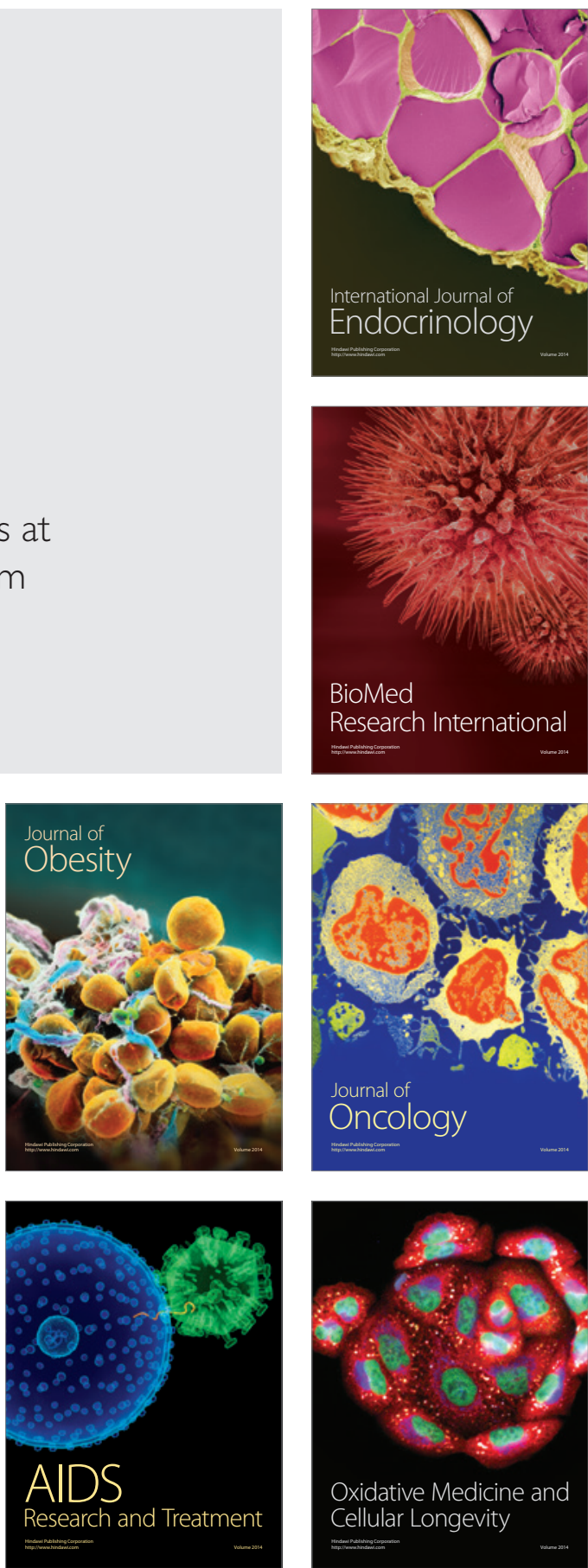